\title{
Diagnosis and Management of Wooden Foreign Bodies in the Orbit: A Case Report
}

\author{
Hyo Bong Kim (D), Hoon Kim (D), Soo Yeon Lim (D), In Chang Koh (D) \\ Department of Plastic and Reconstructive Surgery, Konyang University Hospital and Myunggok Medical Research Center, Konyang University College of \\ Medicine, Daejeon, Korea
}

\begin{abstract}
The diagnosis and management of intraorbital foreign bodies are challenging for surgeons. Foreign bodies made of wood and those located close to the optic nerve are especially difficult to manage. Herein, we report our experience with the diagnosis and management of intraorbital wooden foreign bodies utilizing magnetic resonance imaging (MRI). A 50-year-old man presented to the emergency room with a laceration on his left upper eyelid. No foreign bodies were visible through the laceration, and computed tomography findings showed only evidence of cellulitis with abscess formation. Despite drainage and antibiotics, the cellulitis worsened; subsequently, we found multiple wooden foreign bodies on MRI. Surgical removal was performed, excluding those in the intraconal space to avoid optic nerve damage. The patient recovered well and has not experienced any ophthalmic complications at 1 year of follow-up.
\end{abstract}

Keywords: Foreign body; Orbit; Eye foreign object

\section{Introduction}

Intraorbital foreign bodies often cause serious complications, such as inflammation, abscess, impaired eye movement, and blindness [1]. The degree of injuries depends on the type, size, and location of the foreign body [2]. In particular, unidentified intraorbital foreign bodies that penetrate through external wounds and those made of organic materials, such as wood, are the most challenging for surgeons. The presence of organic intraorbital material, because of its radiolucent character, may be difficult to confirm through imaging studies [3]. Therefore, in such cases, delayed diagnosis and treatment can lead to clinical aggravation. We present a case report on the diagnosis and management of intraorbital wooden foreign bodies. Although the wooden foreign bodies could not be confirmed through external wound and computed tomography (CT) findings, they could be diagnosed using magnetic resonance imaging (MRI) owing to the hypointensity of wood [3,4]. Contrary to previously reported cases, in our case, we decided not to remove all the foreign bodies in the intraconal space, since their removal would have inevitably caused optic nerve damage. We instead removed the wooden foreign bodies in the extraconal space and decided to follow a conservative approach using antibiotics. We obtained good outcomes without serious complications throughout the 1-year follow-up. The patient provided informed consent, and the study design was approved by the Konyang University Hospital Institutional Review Board (IRB No. 2020-12-022).

\section{Case Report}

Received: March 10, 2021

Revised: May 25, 2021

Accepted: May 26, 2021

Corresponding author: In Chang Koh, M.D., Ph.D.

Department of Plastic and Reconstructive Surgery, Konyang University Hospital and Myunggok Medical Research Center, Konyang University College of Medicine, 158

Gwanjeodong-ro, Seo-gu, Daejeon 35365,

Korea

Tel: +82-42-612-2350

Fax: +82-42-600-6057

E-mail: inns@naver.com

This is an Open Access article distributed under the terms of the Creative Commons Attribution Non-Commercial License (https://creativecommons.org/licenses/by-nc/4.0/) which permits unrestricted non-commercial use, distribution, and reproduction in any medium, provided the original work is properly cited.

(C) 2021 Korean Wound Management Society 


\section{Case}

A 50-year-old man presented to the emergency room with a 2.5-cm laceration in the left upper eyelid and periorbital swelling. Owing to alcohol intoxication, the patient could not remember the details of the accident. He only stated that he had fallen in the countryside. Physical examination confirmed that the pupil size, light reflex, visual acuity, and extraocular movements (EOM) were intact; a subconjunctival hemorrhage was observed. After CT scanning and an ophthalmology consultation, a retrobulbar hemorrhage with emphysema was diagnosed (Fig. 1A). The clinical symptoms were presumed to be related to simple traumatic swelling because foreign bodies were not visible on the CT images or on exploration of the eyelid laceration under local anesthesia.

The patient was prescribed oral antibiotics, and returned for outpatient follow-up 2 days later. Serious periorbital swelling
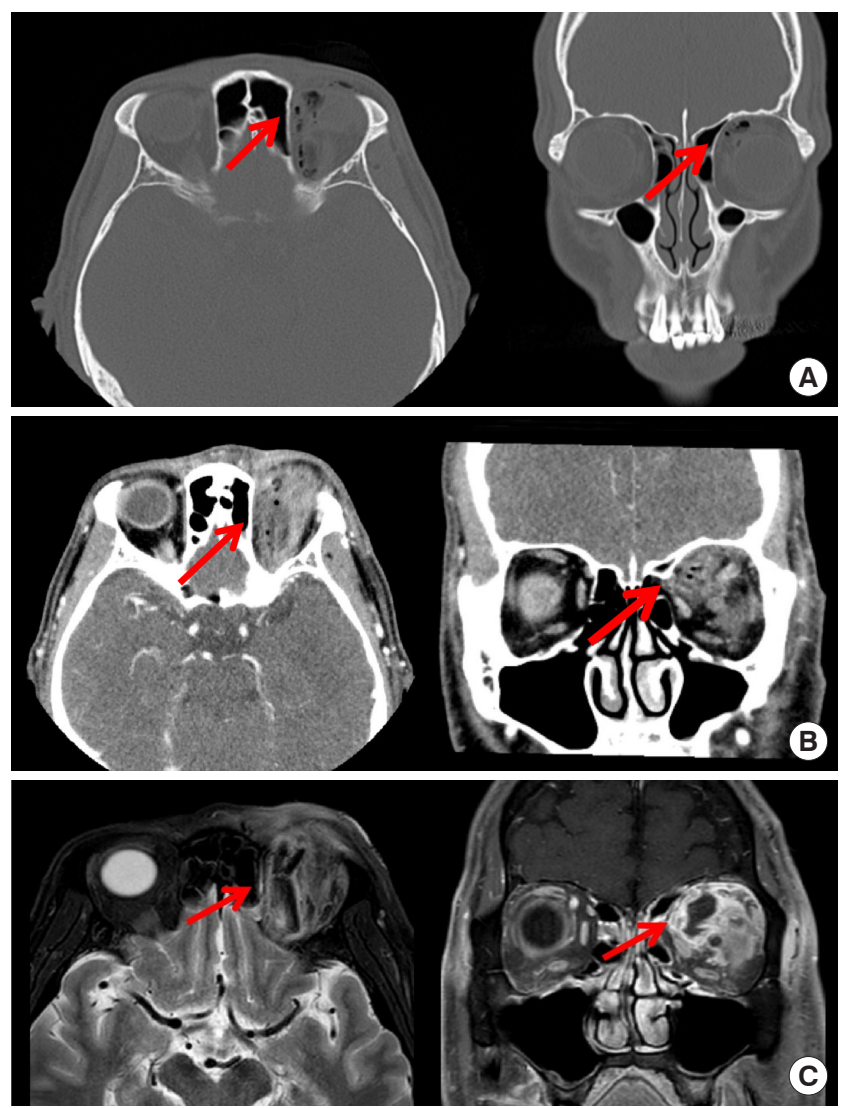

Fig. 1. Preoperative computed tomography and magnetic resonance images (first surgery). (A) Wooden foreign body (red arrow) interpreted as emphysema by the ophthalmologist and radiologist. (B) Aggravation of orbital cellulitis without detection of wooden foreign bodies (red arrow). (C) Wooden foreign bodies (red arrow) in the extraconal and intraconal spaces. and pus discharge were observed, and the patient was admitted for intravenous antibiotics and incisional drainage (Fig. 2). Enhanced CT images for follow-up revealed no foreign bodies in the orbit; however, emphysema and cellulitis with abscess formation were noted (Fig. 1B). Pantoea agglomerans and Enterobacter species, commonly isolated from plants or soil, were identified on a pus swab culture. However, in spite of drainage and antibiotic treatment, the condition worsened. After excluding the presence of metallic foreign bodies through CT scanning, wooden foreign body infection was suspected based on the condition of trauma, pus swab culture and emphysema-like findings of initial and follow-up CT scans. An MRI of the orbit was performed, revealing intraorbital wooden foreign bodies in the intraconal and extraconal spaces (Fig. 1C). Subsequently, we performed surgical exploration under general anesthesia and removed multiple wooden foreign bodies through a previous upper eyelid wound. The largest wooden piece was approximately $3.5-\mathrm{cm}$ long. Despite the removal of multiple foreign bodies from the orbit, followup MRI showed remnant foreign bodies in the extraconal and intraconal spaces (Fig. 3). With the patient's consent, a second operation was performed to remove the remaining foreign bodies (Fig. 4). Postoperative MRI showed no foreign body in the extraconal spaces; however, a few small foreign bodies re-

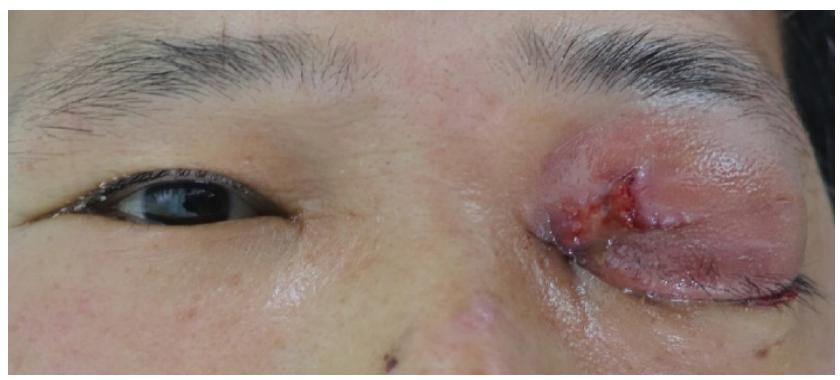

Fig. 2. Preoperative clinical photo. Orbital cellulitis with abscess formation and severe ptosis.

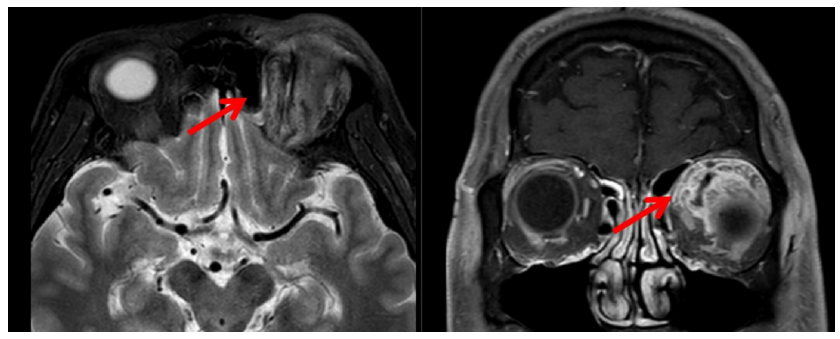

Fig. 3. Postoperative magnetic resonance images (first surgery). Remaining wooden foreign bodies (red arrow) in the extraconal and intraconal spaces. 


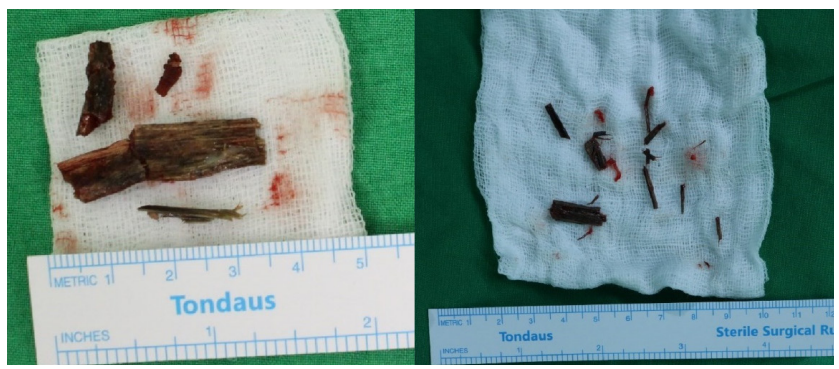

Fig. 4. Wooden foreign bodies retrieved from the orbit.
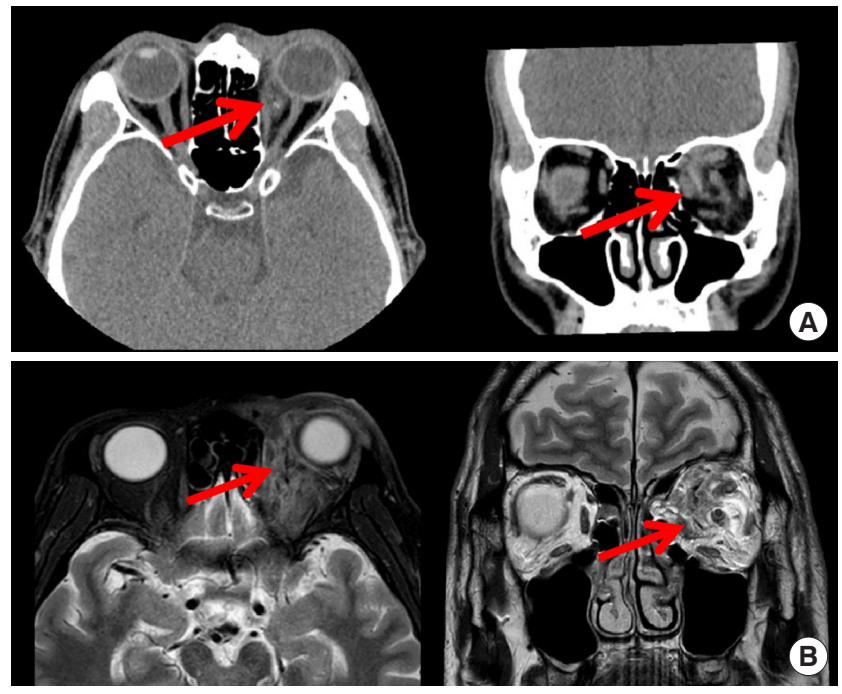

Fig. 5. Postoperative computed tomography and magnetic resonance images (second surgery). (A, B) Remaining wooden foreign bodies (red arrow) in the intraconal space.

mained in the intraconal space, in which the optic nerve is located (Fig. 5). After ophthalmological and radiological consultation, and considering the risks and benefits of surgical intervention, we decided on a conservative approach with close clinical observation, after explaining to the patient that encapsulation of the remnant foreign bodies was expected. The cellulitis and abscess resolved, and the patient was discharged without serious ophthalmic complications (Fig. 6). The patient was satisfied with the functional outcome even on follow-up after 1 year.

\section{Discussion}

The incidence of intraorbital foreign bodies in orbital trauma is rare (2.9\%) [5]. Based on their chemical composition, intraorbital foreign bodies can be divided into (1) metal, (2) nonmetal like glass, plastic, and (3) organic materials (such as

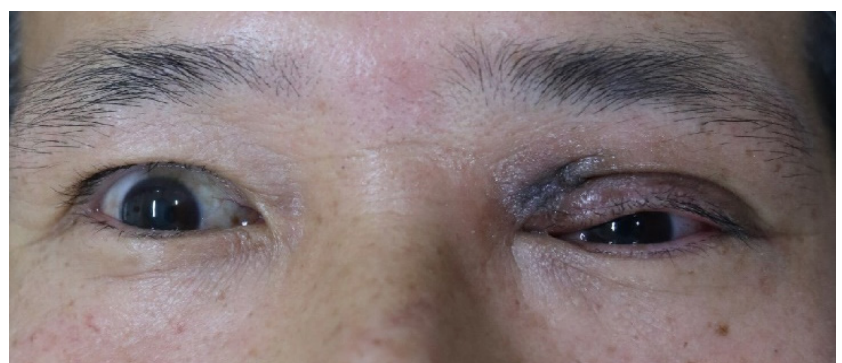

Fig. 6. Clinical photograph taken at the 1-month follow-up. It shows satisfactory recovery without serious complications other than mild ptosis.

wood) [2]. Foreign bodies are mainly diagnosed by imaging studies. Metal and glass are visible on plain radiography and CT due to their radiopaque nature; however, plastic or wood are not visible owing to their radiolucent nature. Therefore, further evaluations such as MRI are required to diagnose wooden foreign bodies and thus, the final diagnosis may be delayed. Wooden foreign bodies may result in severe inflammation because of their porosity, which makes them prone to cause microbial infection [6]. Infection may result in complications such as orbital cellulitis, abscess formation, and brain abscesses [1]. Therefore, literature suggests the diagnosis and removal of all organic foreign bodies as soon as possible if the surgical area is adequate and the patient is willing [7].

In our case, the traumatic event was unclear, and the wooden foreign bodies were not visible through the laceration. In the emergency room, the patient had no prominent symptoms, and an ophthalmologist and a radiologist interpreted the CT scan findings as emphysema. Clinical aggravation, despite antibiotic treatment and incisional drainage, led to suspicion of intraorbital foreign bodies. Based on the patient's inaccurate statement and pus swab culture results, we were eventually able to suspect wooden foreign bodies and confirmed our diagnosis through MRI [8]. Wooden foreign bodies are invisible to plain radiography due to their radiolucent nature and are prone to be misdiagnosed as emphysema on CT [3,4]. In the acute period, wooden foreign bodies show a low density, similar to air bubbles, on CT findings [3,4]. MRI may be better at detecting wooden foreign bodies due to the hypointensity of wood that contrasts with the intraorbital fat on both $\mathrm{T} 1$ and T2-weighted sequences [3,4]. However, MRI should be avoided if metallic foreign bodies have not been excluded because of the possibility of migration $[3,4]$. Therefore, conditions that alert the possibility of intraorbital foreign bodies should be recognized, such as the exact mechanism of trauma, clinical 
aggravation, and EOM limitation [9]. Moreover, ultrasound may be an alternative since wooden foreign bodies are characterized by hyperechoic structures with posterior shadowing on the ultrasound image. In the case of wooden foreign bodies, pus swab cultures may be useful, and imaging modalities appropriate for the diagnosis should be sequentially selected.

In previously reported cases of wooden intraorbital foreign bodies, good outcomes were obtained through a surgical approach with the knowledge of the exact number and location of intraorbital foreign bodies $[7,10]$. However, surgical approaches may not be favorable if postoperative complications, such as optic nerve injury, are anticipated due to the location of the foreign body in the intraconal space being very close to the optic nerve. Unlike in previously reported cases, we chose a conservative approach with close clinical observation following removal of foreign bodies in the extraconal space. Since the intraconal foreign body removal would have inevitably damaged the optic nerve, residual foreign body encapsulation could be expected using antibiotics to control inflammation. The patient improved without serious ophthalmic complications and showed good progress without the need for additional surgery. On a 1-year follow-up, the patient maintained good outcome without serious ophthalmic complications except for mild diplopia on medial gaze. Meticulous intraorbital foreign body removal is important to prevent complications, and conservative care may be a good option when severe optic nerve damage is expected. The follow-up period was relatively short, which could be a limitation of our study. Thus, we will continue to observe the patient for possible complications such as intraorbital abscess, visual loss, and limitation of eyeball motion caused by delayed foreign body reactions.

In conclusion, appropriate diagnosis and treatment can minimize complications in cases of orbital wooden foreign bodies, and conservative treatment can be considered if optic nerve damage is inevitable.

\section{Conflict of interest}

No potential conflict of interest relevant to this article was reported.

\section{ORCID iDs}

Hyo Bong Kim

https://orcid.org/0000-0002-2256-0784

Hoon Kim

Soo Yeon Lim

In Chang Koh

https://orcid.org/0000-0003-1261-9743

https://orcid.org/0000-0002-2954-2191

https://orcid.org/0000-0002-5902-5670

\section{References}

1. Fulcher TP, McNab AA, Sullivan TJ. Clinical features and management of intraorbital foreign bodies. Ophthalmology 2002;109:494-500.

2. Ananth Kumar GB, Dhupar V, Akkara F, et al. Foreign body in the orbital floor: a case report. J Maxillofac Oral Surg 2015;14:832-5.

3. Nagae LM, Katowitz WR, Bilaniuk LT, et al. Radiological detection of intraorbital wooden foreign bodies. Pediatr Emerg Care 2011;27:895-6.

4. Ho VT, McGuckin JF Jr, Smergel EM. Intraorbital wooden foreign body: CT and MR appearance. AJNR Am J Neuroradiol 1996;17:134-6.

5. Bartkowski SB, Kurek M, Stypulkowska J, et al. Foreign bodies in the orbit: review of 20 cases. J Maxillofac Surg 1984;12:97-102.

6. Sitaula S, Gautam V. Intraorbital wooden foreign body: a case report. J Kathmandu Med Coll 2015;3:119-21.

7. Gonullu ME, Filinte GT, Cardak NG, et al. The surgical strategy for the intraorbital foreign bodies. J Craniofac Surg 2016;27:1785-8.

8. Dunn IF, Kim DH, Rubin PA, et al. Orbitocranial wooden foreign body: a pre-, intra-, and postoperative chronicle: case report. Neurosurgery 2009;65:E383-4.

9. Santos Tde S, Melo AR, Moraes HH, et al. Impacted foreign bodies in orbital region: review of nine cases. Arq Bras Oftalmol 2010;73:438-42.

10. Kim YH, Kim H, Yoon ES. Unrecognized intraorbital wooden foreign body. Arch Craniofac Surg 2018;19:300-3. 